\title{
Different attachment styles correlate with mood disorders in adults with epilepsy or migraine
}

${ }^{1,2}$ Marco Mula, ${ }^{1}$ Davies Danquah-Boateng, ${ }^{1,2}$ Hannah R Cock, ${ }^{2,3}$ Usman Khan, ${ }^{1,2}$ Dora A. Lozsadi, ${ }^{2,3}$ Niranjanan Nirmalananthan

${ }^{1}$ Epilepsy Group, Atkinson Morley Regional Neuroscience Centre, St George's University Hospitals NHS Foundation Trust, London, United Kingdom

${ }^{2}$ Institute of Medical and Biomedical Sciences, St George's University of London, United Kingdom

${ }^{3}$ Headache Group, Atkinson Morley Regional Neuroscience Centre, St George's University Hospitals NHS Foundation Trust, London, United Kingdom

\section{Corresponding author:}

Dr Marco Mula MRCP London MD PhD

Epilepsy Group

Atkinson Morley Regional Neuroscience Centre

St George's University Hospitals NHS Foundation Trust

Blackshaw Road

London SW17 0QT

United Kingdom

Tel: +442087254107

FAX: +442087254591

Email: mmula@sgul.ac.uk 


\section{ABSTRACT}

Purpose: Interpersonal relationships are viewed as important contexts within which psychopathology emerges and persists or desists. Attachment theory describes the dynamics of longterm relationships between humans especially as in families and life-long friendships. The present study is aimed at investigating attachment styles in adult patients with epilepsy as compared to subjects with migraine and their potential correlates with a history of mood disorders.

Methods: A consecutive sample of 219 adult outpatients with epilepsy (117) or migraine (102) has been assessed with the Attachment Style Questionnaire (ASQ).

Results: Patients with epilepsy and a lifetime history of mood disorders presented elevated scores for Need for approval $(\mathrm{p}<0.001)$ and Preoccupation with relationships $(\mathrm{p}<0.001)$. Age correlated with Relationships as secondary $(r=0.322 ; p<0.001)$ and Need for approval $(r=0.217 ; p=0.019)$ subscales while age at onset correlated only with Relationships as secondary $(r=0.225 ; p=0.015)$. Seizure free patients presented lower scores for Need for approval $(p=0.003)$. Patients with migraine and a lifetime history of mood disorders presented lower scores in Confidence $(p=0.002)$ and higher scores in Discomfort with closeness ( $\mathrm{p}=0.026)$.

Conclusions: An anxious-preoccupied attachment correlates with mood disorders in epilepsy while an avoidant pattern in migraine. Our results bring further data on the role of psychological variables for mood disorders in epilepsy. Further studies will allow early identification of patients at risk and the development of preventive strategies.

Key words: epilepsy, depression, migraine, attachment, personality 


\section{INTRODUCTION}

Mood disorders represent a frequently encountered psychiatric comorbidity in patients with epilepsy whose determinants are both biological and psychosocial [1]. They significantly increase morbidity and mortality [2], being an important predictor of low quality of life (QoL) [3], treatmentemergent adverse events of antiepileptic drugs [4] and poor response to AED treatment [5]. Among psychosocial variables, several authors have pointed out the role of stigma, discrimination [6], locus of control, attributional style, adjustment to epilepsy [7], socioeconomic status, social support and parental overprotection [8]. The identification of psychological factors has relevant implications in terms of developing specific strategies of prevention and treatment. Despite the number of studies on stigma and social determinants in epilepsy, no studies investigated the role of interpersonal relationships as a potential clinical correlate for mood disorders in epilepsy. However, it is evident that interpersonal relationships are pivotal for studying psychopathology in general and when there is a psychological disturbance, interpersonal relationships also are likely to be disturbed, given the critical importance of relationships in human adaptation. In fact, social relationships are viewed as important contexts within which psychopathology emerges and persists or desists. Attachment theory describes the dynamics of long-term relationships between humans especially as in families and life-long friendships. Its most important tenet is that an infant needs to develop a relationship with at least one primary caregiver for social and emotional development to occur normally, and that further relationships build on the patterns developed in the first relationship [9]. Initially developed by the English psychotherapist John Bowlby [10], it has been subsequently reinforced by Mary Ainsworth [11] who theorized three specific attachment patterns: secure, avoidant and anxious attachment. A fourth pattern, disorganized attachment, was identified later [12]. According to these authors, experiences with attachment figures give rise to representational or "working models" called attachment styles that guide behaviour, affect and perception in relationships. Attachment styles are regarded as pre-programmed schemes which appear and develop with the aim of permitting closeness and contact with the mother figure and therefore, biologically speaking, increasing the probability of surviving. These models, to a certain extent based on some specific innate capacities, are modified and organized through environmental influences and result in more complex and hierarchically organized systems made of tactics and strategies. It seems, thus, obvious that the diagnosis of a chronic disease with a significant psychological burden may further shape the final pattern. Furthermore, attachment theory has been recently revitalized by a number of studies suggesting a neurobiological basis for attachment with oxytocin being a major biochemical mediator [13]. In this perspective, it is tempting to speculate that different brain disorders may further interact with the underlying attachment pattern in the final development of psychopathology.

The present study is aimed at describing attachment styles in adults with epilepsy as compared to subjects with migraine and at investigating their potential correlates with a history of mood disorders.

\section{METHODS}

A consecutive sample of adult outpatients with epilepsy or migraine attending the Neurology Outpatient Department, St George's University Hospitals, were invited to participate in the study. Eligible patients included new and continuing patients more than 18 years of age; either an established diagnosis of epilepsy according to the International League Against Epilepsy (ILAE) criteria or an established diagnosis of migraine according to the International Headache Society (IHS) criteria; be willing to provide written and witnessed informed consent; have no gross cognitive deficits and a sufficient reading ability to manage the questionnaire. This study has been reviewed and given a favourable opinion by the National Research Ethics Service Committee, London - South East. Eligible subjects provided written informed consent, after receiving a complete description of the study and having the opportunity to ask questions. 
Clinical and demographic data, as well as lifetime and family psychiatric history were collected from paper and electronic notes using a standardised form. All subjects were assessed with the Attachment Style Questionnaire (ASQ) [14]. The ASQ is a 40-item, self-report questionnaire, specifically developed for adult subjects to rate aspects of themselves and others on a 6-point Likert scale. The instrument is focused on relationships in general rather than romantic or close relationships. It has a lifetime perspective as attachment by definition is an innate behavioural pattern. The ASQ yields five subscales: Confidence, Discomfort with closeness, Relationships as secondary, Need for approval, Preoccupation with relationships. The ASQ showed high internal consistency and good reliability [14]. Itis available in several languages and has been used in hundreds of studies on this subject.

Frequencies of categorical demographic and clinical variables were analysed using the $\chi 2$ analysis or Fisher's exact test. Continuous demographic and clinical variables as well as ASQ scores were compared using the Student's t-test for independent samples. Correlations were tested using a bivariate two-tailed parametric correlation procedure (Pearson's coefficient). A logistic regression model was then constructed for lifetime history of mood disorders using the identified ASQ subscale scores and with age and gender as potential confounders. The alpha error was set at 0.05 . All statistical analyses were 2-tailed and conducted using the Statistical Package for Social Sciences (Version 15 for Windows, SPSS Inc. Chicago, IL).

\section{RESULTS}

A total of 219 patients were assessed, 117 with epilepsy and 102 with migraine. The two groups differed in terms of gender and education level with more females and a higher education level in the migraine group as compared to the epilepsy group (Table 1). There was no difference in terms of lifetime history of mood disorders between the groups and in both cases there was a high comorbidity with anxiety disorders (Table 2 ). Only two patients had a previous history of psychosis in the epilepsy group and none in the migraine group. There was an increased family history of psychiatric problems in patients with epilepsy as compared to the migraine group ( $47.9 \%$ vs. $27.5 \%$ $\mathrm{p}<0.001)$.

There was no difference in ASQ scores between patients with epilepsy and migraine. There was no gender difference in ASQ scores in both groups. There was no difference in ASQ scores comparing patients with and without a family history of psychiatric disorders.

Epilepsy group: There was no relationship between ASQ scores and epilepsy syndrome (Focal vs. Generalised), localisation (Temporal Lobe Epilepsy vs. ExtraTemporal), laterality (Dominant vs. NonDominant). Patients with a lifetime history of mood disorders presented elevated scores for Need for approval $(\mathrm{t}=4.279 \mathrm{p}<0.001)$ and Preoccupation with relationships $(\mathrm{t}=1.137 \mathrm{p}<0.001)$ subscales (Fig. 1). In patients with epilepsy and mood disorders, age correlated with Relationships as secondary ( $r=$ $0.322 ; p<0.001)$ and Need for approval $(r=0.217 ; p=0.019)$ subscales while age at onset correlated only with Relationships as secondary $(r=0.225 ; p=0.015)$. Seizure free patients presented lower scores for Need for approval compared to non-seizure free (Free $19.9+/-7.0$ vs. No Free $24.0+/-7.5$; $t=-$ 3.082; $\mathrm{p}=0.003$ ).

Migraine group: There was no relationship between ASQ scores and migraine type (Episodic vs. Chronic). There was no relationship with presence/absence of Medication Overuse Headache (MOH). There were no correlations between age or age at onset and ASQ scores. Patients with a lifetime history of mood disorders presented lower scores in Confidence $(t=-3287 \mathrm{p}=0.002)$ and higher scores in Discomfort with closeness ( $\mathrm{t}=2.260 \mathrm{p}=0.026)$ (Fig. 1).

\section{DISCUSSION}


Our results show that an insecure attachment style correlates with a lifetime history of mood disorders in patients with epilepsy or migraine and this is in keeping with recent literature suggesting that an insecure attachment is associated with an increased risk of psychopathology, especially mood disorders [15]. Our data clearly brings further information on the role of psychological variables for mood disorders in epilepsy and, interestingly enough, seems to suggest that different attachment patterns are implicated in epilepsy and migraine regarding comorbid mood disorders. Someone could argue that the observed difference is related to the different distribution of gender and educational level in the two groups. However, there were no gender-related differences in ASQ scores in our population. In addition, the relationship between gender and attachment is still controversial. In fact, it is a common stereotype of Western culture that men are more emotionally distant and dismissing than women [16] and empirical studies have tended to substantiate this portrayal of men only in the context of romantic relationships but not for attachment in general. In fact, the majority of authors seem to agree that men and women display similar attachment styles and similarities are even more evident in cultures with high mortality, few resources and high fertility rates [17]. Regarding different educational levels, it seems established that educational level and socioeconomic status are not determinants for attachment style [18]. Therefore, the different attachment styles observed in the two groups regarding comorbid mood disorders seem to be due to genuine underlying differences.

Patients with epilepsy and mood disorders present with high scores for Need for approval and Preoccupation with relationships. Both factors mirror the anxious-preoccupied attachment style. These subjects seek high levels of intimacy, approval and responsiveness from partners or relatives, becoming overly dependent. According to the original theory of Bowlby, this behaviour is developed when the caregiver selectively chooses when to attend to the child, often being absent in times of need [10]. The anxious child is uncertain whether, in time of need, the primary caregiver will come to his or her aid, and as a result, the child will also present separation anxiety and will be unwilling and anxious to try and experience new things. In such a perspective, it is easy to understand the potential impact of a disease like epilepsy characterised by highly unpredictable events like seizures. It is, thus, tempting to speculate that subjects with an anxious-preoccupied attachment are less able to cope with epilepsy than those with a secure attachment and consequently are at increased risk of depression. Our data does not allow us to confirm that an anxious-preoccupied attachment style is associated with depression following the diagnosis of epilepsy because the onset of depression was not recorded but the observed difference in Need for approval scores in seizure free patients further suggests that epilepsy per se emphasises dependent aspects in preoccupied individuals. Obviously, this hypothesis would explain mainly depression following epilepsy and not vice versa but investigating the relationship among attachment style, coping strategies and depression, may probably allow the early identification, among newly diagnosed patients, of those in need of further psychological support and the development of specific strategies of prevention for depression.

The correlation of both age and age at onset with the ASQ subscale Relationships as secondary represents another interesting element. This subscale mirrors what Bartholomew and Horowitz defined as the "dismissing domain" of attachment [19] characterised by high levels of avoidant behaviours. Combined with the previously identified elements, this finding suggests that these patients are anxious about being rejected but at the same time, because they tend to avoid intimacy, they have difficulties in compensating for this anxiety. The direct correlation with both age and age of onset suggests that when the epilepsy starts later on in life can have a more disabling impact in subjects with a preoccupied attachment, increasing the risk of developing a mood disorder.

Literature about attachment in headache is definitely more robust than in patients with epilepsy. Berry and Drummond [20] speculated that anxiously attached individuals may express greater pain or may show a stronger attentional bias toward painful sensations than securely attached ones increasing the likelihood of developing headache per se. Our results are in keeping with authors suggesting that an avoidant attachment may represent a risk factor for 
psychopathology in patients with migraine [21-22]. In fact, our subjects present high scores for Discomfort with closeness and low scores for Confidence. The Discomfort with closeness factor mirrors the conceptualization of avoidant attachment given by Hazan and Shaver [23]. These subjects find it difficult to trust others and feel uncomfortable letting others into their lives. High levels in Discomfort with closeness and low levels in Confidence are typical of individuals who don't actively seek interpersonal relationships and tend to live alone, without any form of social/emotional support. Although associated with a number of psychiatric conditions including mood disorders, such an attachment pattern is typical of patients with personality disorders [10].

The interesting point made by our results is that the two identified attachment styles, as correlates of mood disorders in epilepsy and migraine, are somehow opposed. A subject with an anxiouspreoccupied attachment has a low self-esteem and highly regards others, while the avoidant has a high self-esteem and does not seek interpersonal relationships because they poorly regard others. Obviously, both of them are insecure attachments and, as such, predispose to psychopathology but the fact that mood disorders correlate to such a different attachment pattern in these two neurological conditions clearly confirms the role of the disease in giving rise to comorbid depression in different ways in diverse susceptible individuals. This is nicely shown by a regression model including identified ASQ subscale scores, gender and age in the two groups. In epilepsy, male gender is a protective factor while elevated Need for approval scores are associated with an increased risk of lifetime mood disorder in epilepsy. In patients with migraine, only Confident scores are inversely correlated with lifetime mood disorders (Table 3).

Our results should be considered keeping in mind the following limitations. First, a lifetime history of mood disorders was identified from patients' notes and this is not as accurate as a formal psychiatric assessment using, for example, the Structured Interview for DSM Axis I. However, this was a preliminary investigation looking at the potential role of attachment as a psychological determinant for mood disorders in epilepsy. Our results clearly suggest that further studies are needed and that attachment theory provides a useful theoretical framework for integrating research findings from several fields concerning the development of mood disorders in epilepsy and for planning therapeutic interventions. Second, our results may not be representative of the general population of patients with epilepsy as they come from a tertiary referral centre where more severe and drug-refractory cases are present.

In conclusion, patients with epilepsy and mood disorders usually present an underlying anxious-preoccupied attachment and this seems to be typical only of patients with epilepsy. These patients are probably less able to cope with the unpredictability of seizures and the psychological burden associated with the diagnosis of epilepsy. Whether this factor interacts with poor coping strategies should be the focus of future investigations in order to identify, at an early stage, patients at increased risk of depression.

\section{ACKNOWLEDGMENTS AND DISCLOSURE OF CONFLICTS OF INTEREST}

No conflicts of interest with the paper in question. MM is currently serving as Associate Editor of Epilepsy \& Behavior. The authors thankfully acknowledge Ms Anne Marie Logan (Headache Practitioner), Ms Amanda Reeve and Ms Marie Synnott-Wells (Epilepsy Clinical Nurse Specialist). The authors are grateful to St George's University of London and St George's University Hospitals NHS Foundation Trust for their support.

\section{REFERENCES}

1. Kanner AM. Depression and epilepsy: a review of multiple facets of their close relation. Neurol Clin 2009;27: 865-80. 
2. Mula M, Schmitz B, Sander JW. The pharmacological treatment of depression in adults with epilepsy. Expert Opin Pharmacother. 2008;9:3159-68.

3. Boylan LS, Flint LA, Labovitz DL, Jackson SC, Starner K, Devinsky O. Depression but not seizure frequency predicts quality of life in treatment-resistant epilepsy. Neurology. 2004;62:258-61.

4. Mula M, Sander JW. Negative effects of antiepileptic drugs on mood in patients with epilepsy. Drug Saf. 2007;30:555-67.

5. Hitiris N, Mohanraj R, Norrie J, Brodie MJ. Mortality in epilepsy. Epilepsy Behav. 2007;10:363-76.

6. de Boer HM, Mula M, Sander JW. The global burden and stigma of epilepsy. Epilepsy Behav 2008;12: 540-6.

7. Goldstein LH, Holland L, Soteriou H, Mellers JD. Illness representations, coping styles and mood in adults with epilepsy. Epilepsy Res. 2005;67:1-11

8. Lambert MV, Robertson MM. Depression in epilepsy: etiology, phenomenology, and treatment. Epilepsia 1999; 40(Suppl 10):S21-47.

9. Richer Ed. The Clinical Applications of Ethology and Attachment Theory. ACAMH Occasional Papers No.9. London: Association for Child and Adolescent Mental Health

10. Bowlby J. Attachment and loss: Vol. I. Attachment. New York, Basic Books. 1969/1982

11. Ainsworth MD, Blehar MC, Waters E, Wall S. Patterns of attachment: a psychological study of the strange situation. Hillsdale, NJ: Lawrence Erlbaum Associates, Inc; 1978.

12. Vaughn BE, Lefever GB, Seifer R, Barglow P. Attachment behavior, attachment security, and temperament during infancy. Child Dev. 1989;60:728-37.

13. Costa B, Pini S, Gabelloni P, Abelli M, Lari L, Cardini A, Muti M, Gesi C, Landi S, Galderisi S, Mucci A, Lucacchini A, Cassano GB, Martini C. Oxytocin receptor polymorphisms and adult attachment style in patients with depression. Psychoneuroendocrinology. 2009 Nov;34(10):1506-14.

14. Feeney JA, Noller P \& Hanrahan M. Assessing adult attachment. In: MB Sperling, WH Berman (eds), Attachment in adults: clinical and developmental perspectives. New York, Guilford Press 1994:122-158.

15. Dozier M, Stovall KC, Albus KE. Attachment and psychopathology in adulthood. In: Shaver PR, Cassidy J (eds). Handbook of attachment: theory, research, and clinical applications. New York, Guilford Press 1999:497-519.

16. Bem SL. The lenses of gender. New Haven, Yale University Press 1993

17. Costa PT Jr, Terracciano A, McCrae RR. Gender differences in personality traits across cultures: robust and surprising findings. J Pers Soc Psychol. 2001;81:322-31

18. Simpson JA. Attachment theory in modern evolutionary perspective. In: Cassidy J, Shaver PR (Eds.). Handbook of attachment. New York: Guilford 1999:115-140

19. Bartholomew K, Horowitz LM. Attachment styles among young adults: a test of a four-category model. J Pers Soc Psychol 1991;61:226-244

20. Berry JK, Drummond PD. Does attachment anxiety increase vulnerability to headache? J Psychosom Res. 2014;76:113-20.

21. Esposito M, Parisi L, Gallai B, Marotta R, Di Dona A, Lavano SM, Roccella M, Carotenuto M. Attachment styles in children affected by migraine without aura. Neuropsychiatr Dis Treat. 2013;9:1513-9.

22. Savi L, Buccheri R, Tambornini A, De Martino P, Albasi C, Pinessi L. Attachment styles and headache. J Headache Pain. 2005;6:254-7.

23. Hazan C, Shaver P. Romantic love conceptualized as an attachment process. J Pers Soc Psychol 1987;52: 511-524. 
Table 1. Demographic and clinical characteristics of the study sample (total sample $N=219$ )

\begin{tabular}{|c|c|c|c|}
\hline & $\begin{array}{c}\text { Epilepsy } \\
\mathrm{N}=\mathbf{1 1 7}(\%)\end{array}$ & $\begin{array}{c}\text { Migraine } \\
\mathrm{N}=102(\%)\end{array}$ & Test; 2-tailed P value \\
\hline \multicolumn{4}{|l|}{ Gender } \\
\hline Males & $59(50.4)$ & $21(20.6)$ & Chi Square $=20.927 ; p<0.001$ \\
\hline Female & $58(49.6)$ & $81(79.4)$ & \\
\hline Age, years (SD) & $41.4(14.7)$ & $41.1(13.7)$ & n.s. \\
\hline \multicolumn{4}{|l|}{ Education } \\
\hline Primary School & $12(10.3 \%)$ & $5(4.9 \%)$ & Chi Square $=14.511 ; p=0.013$ \\
\hline High school & $42(35.9 \%)$ & $36(35.3 \%)$ & \\
\hline University degree & $18(15.4 \%)$ & $33(32.4 \%)^{*}$ & \\
\hline Post-doctoral & $4(3.4 \%)$ & $7(6.9 \%)$ & \\
\hline \multicolumn{4}{|l|}{ Working situation } \\
\hline Employed & $68(58.1 \%)$ & $67(65.6 \%)$ & n.s. \\
\hline Student & $5(4.3 \%)$ & $5(4.9 \%)$ & \\
\hline Unemployed & $27(23.1 \%)$ & $28(27.5 \%)$ & \\
\hline Retired & $17(14.5 \%)$ & $2(2 \%)$ & \\
\hline Age at onset of the disease, years (SD) & $26.8(16.9)$ & $25.9(14.5)$ & n.s. \\
\hline \multicolumn{4}{|c|}{ Epilepsy type } \\
\hline Focal & 79 (67.5\%) & - & \\
\hline Generalized & $24(20.5 \%)$ & - & \\
\hline Undetermined & $14(12 \%)$ & - & \\
\hline \multicolumn{4}{|l|}{ Aetiology } \\
\hline Unknown & $63(53.8 \%)$ & - & \\
\hline Structural/metabolic & $54(46.2 \%)$ & - & \\
\hline \multicolumn{4}{|l|}{ Seizure frequency } \\
\hline Free & $61(52.1 \%)$ & - & \\
\hline 1-10 / year & $32(27.4 \%)$ & - & \\
\hline $1-10 /$ month & $15(12.8 \%)$ & - & \\
\hline $11-20 /$ month & $4(3.4 \%)$ & - & \\
\hline$>20 /$ month & $5(4.3 \%)$ & - & \\
\hline \multicolumn{4}{|l|}{ Therapy } \\
\hline No therapy & $1(0.9 \%)$ & $24(23.5 \%)$ & \\
\hline Monotherapy & $1(69.2 \%)$ & $59(57.8 \%)$ & \\
\hline Dual therapy & $29(24.8 \%)$ & $13(12.7 \%)$ & \\
\hline Polytherapy & $6(5.1 \%)$ & 6 (5.9\%) & \\
\hline \multicolumn{4}{|l|}{ Migraine type } \\
\hline Episodic & - & 29 (28.4\%) & \\
\hline Chronic & - & 73 (71.6\%) & \\
\hline Medication-Overuse Headache & - & $20(19.6 \%)$ & \\
\hline
\end{tabular}


Table 2. Personal and family history of psychiatric disorders and attachment style questionnaire scores in the study sample $(\mathrm{N}=\mathbf{2 1 9})$.

\begin{tabular}{lccc}
\hline & $\begin{array}{c}\text { Epilepsy } \\
\mathbf{N}=\mathbf{1 1 7}\end{array}$ & $\begin{array}{c}\text { Migraine } \\
\mathbf{N}=\mathbf{1 0 2}\end{array}$ & Test, $\mathbf{p}$ value \\
\hline $\begin{array}{l}\text { Lifetime history of mood disorders (\%) } \\
\text { Family history of any psychiatric disorder (\%) }\end{array}$ & $36(30.8 \%)$ & $45(44.1 \%)$ & $\begin{array}{c}\text { n.s. } \\
\end{array}$ \\
& $56(47.9 \%)$ & $28(27.5 \%)$ & $\begin{array}{c}\text { Chi Square }=9.603 \\
\mathrm{p}=0.001\end{array}$ \\
ASQ Confidence (SD) & $33.7(5.8)$ & $33.4(7.6)$ & n.s. \\
ASQ Discomfort with closeness (SD) & $35.0(8.0)$ & $37.3(10)$ & n.s. \\
ASQ Relationships as secondary (SD) & $17.8(6.6)$ & $18.6(6.7)$ & n.s. \\
ASQ Need for approval (SD) & $21.8(7.5)$ & $24.1(7.6)$ & n.s. \\
ASQ Preoccupation with relationships (SD) & $27.7(7.7)$ & $28.3(8.4)$ & n.s. \\
\hline
\end{tabular}

Table 3. Regression model for lifetime history of mood disorders (adjusted for age).

\begin{tabular}{|l|c|c|c|c|}
\hline EPILEPSY & Wald & P value & Exp(B) & 95\%Cl \\
\hline Gender (male) & 13.664 & $<0.001$ & 0.159 & $0.060-0.422$ \\
\hline $\begin{array}{l}\text { ASQ Need for } \\
\text { approval }\end{array}$ & 12.413 & $<0.001$ & 1.124 & $1.053-1.200$ \\
\hline MIGRAINE & & & & \\
\hline ASQ Confidence & 9.925 & 0.002 & 0.905 & $0.850-0.963$ \\
\hline
\end{tabular}

Figure 1. Attachament Style Questionnaire (ASQ) subscale scores in patients with epilepsy (E) or migraine (M) with or without a lifetime history of mood disorders (MD).

${ }^{*} \mathrm{t}=-3287 \mathrm{p}=0.002 ; * * t=2.260 \mathrm{p}=0.026 ; * * * t=4.279 p<0.001 ; * * * * t=1.137 p<0.001$ 